\title{
Software for Modeling Oilfield Surface Equipment Mode
}

\author{
Iakov S. Korovin* and Maxim G. Tkachenko \\ Southern Federal University, Rostov-on-Don \\ Corresponding author
}

\begin{abstract}
In the paper we describe a software developed to simulate the complexes of the surface infrastructure of oil and gas fields. The created software is based on methods and algorithms proposed by the team of authors for constructing models of surface equipment for hydrocarbon deposits. The proposed approaches apply neural network $k$ models of field components, trained on the basis of data from real production sites based on telemetry control data. The interaction between the components of the model is carried out on the basis of modeling the transfer of various types of energy between the components that make up the structure of the model.
\end{abstract}

Keywords-Digital oilfield; neural networks; modeling; oil production.

\section{INTRODUCTION}

A number of works by the author's team [1] described algorithmic approaches to the construction of a complex system based on the use of independent neural network components simulating the operation of surface equipment blocks of a complex for extraction and processing of hydrocarbon raw materials. The library of simulated facilities includes production wells, booster pump station (BPS), pumping equipment of these facilities, pipeline network elements, as well as crude oil preparation complexes for transfer to central collection points. The proposed algorithms are used as neural network methods in modeling the components used, as well as graphical representation of the complex model for combining components into a single interrelated model of the field.

To combine the heterogeneous elements of the model, an approach based on the transmission of unified flows between the model elements is proposed. In the training of neural networks, the energy characteristics of the equipment used, as well as the volumetric mass transfer data (measured by flow and pressure monitoring of the medium) measured at the nodes of the data network are used as these flows.

\section{GENERAL ARCHITECTURE OF THE SOLUTION}

The proposed algorithms were implemented as software in $\mathrm{C}$ ++ programming language in the MS Visual Studio 2012 environment. The software model was prepared to work with actual field data. Neural network training is performed and the settings are loaded into the models of components of the global integrated field model. The software model controls the exchange of data between individual neural networks, which are simulation models of equipment, oil pipeline segments and other components. The controlunit provides transmission of the input and output data of neural networks to each other in accordance with the settings of the block diagram of the integrated model. The block diagram determines the order of motion of the fluid extracted from the well, the fluid passes through the components of the integrated model, partially changing the properties (pressure and temperature changes, component composition). The change in properties consumes energy. The application architecture is schematically shown in Figure 1.

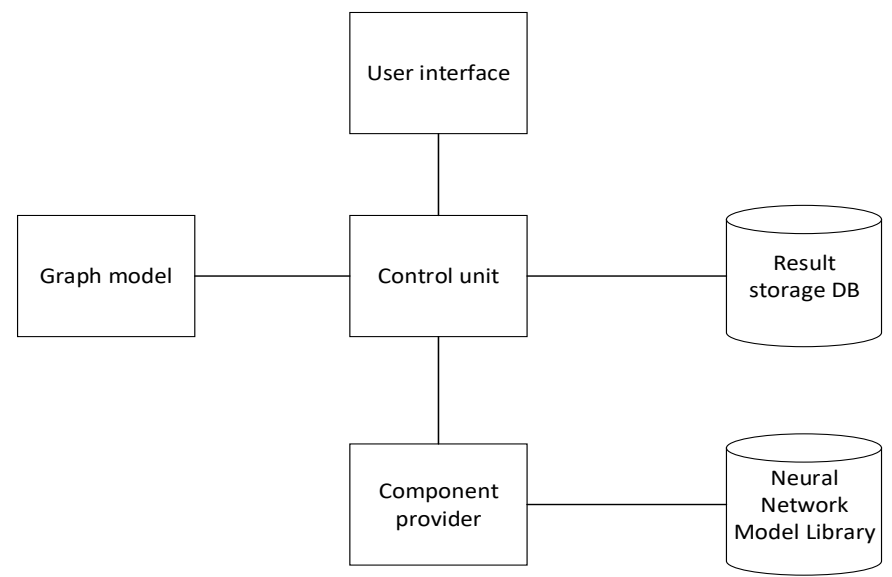

FIGURE I. THE ARCHITECTURE OF THE SOFTWARE MODEL.

To download and edit the initial parametric data for the simulated components, a userinterface has been developed that allows all parameters of the model to be manually changed (Fig. 2).

The results of modeling on individual components of the systemcan be derived both in a tabular form and in the form of graphs (Fig. 3).

The resulted graph shows the results of modeling the pressure loss along the length of the pipeline, for the selected steps. 


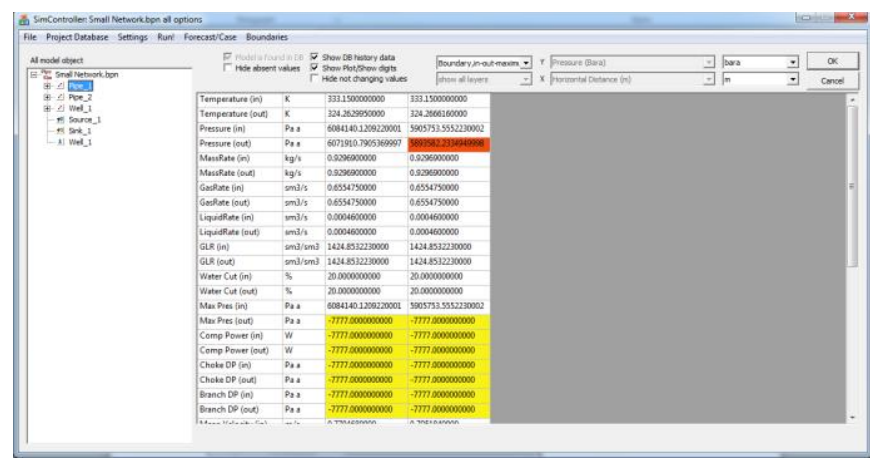

FIGURE II. INTERFACE OF EDITING MODEL PARAMETERS.

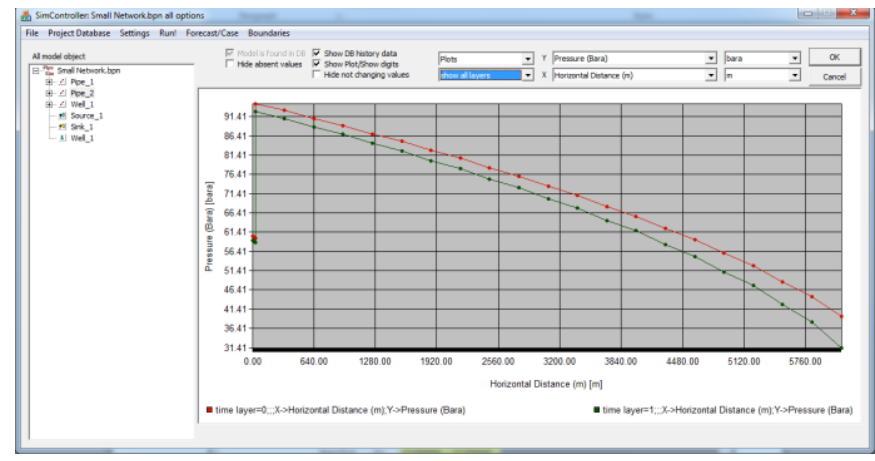

FIGURE III. INTERFACE OF EDITING MODEL PARAMETERS.

\section{INITIAL DAT A FOR MODELING}

The initial data for the program model is determined by the composition of the block diagram of the integrated field model. Each component of the flowchart has an individual set of incoming data, but a similar set of outgoing ones. Incoming data is divided into constants and variables. Constant properties of the block diagram element include design features of the modeled object, such as the diameter of the pipeline, the number of stages of the pump, the efficiency of the electric motor, and others. Variable inputs include the properties of the fluid that enters the flowchart element (pressure, temperature, water cut, free gas volume, dissolved gas volume, density of oil, water, gas). The outgoing data of the flowchart element is also the properties of the fluid that could have changed under the influence of the element, for example, the pressure could have increased if this element is a pump; The gas factor will decrease if this element is a separator.

The database with the results of the calculation receives all data at the intermediate stages of the calculation and outputs the results for viewing through the interface.

The input data for the software model as a whole are prepared in the form of a text file describing the properties of the formation fluid, the construction of the components of the flowchart, and a description of the flowchart in the form of a tree-like description.

The output information of the software model is a collection of records in the database. The results include output information for each of the components of the flowchart, including output information from the last element, which contains information on the final production throughout the field.

\section{MODELING THE SURFACE INFRASTRUCTURE}

The calculations using the integrated model differ from the traditionally performed calculations separately for the hydrodynamic models and separately for the surface infrastructure. With the traditional approach, the production profile is first formed on wells or bushes, and then, under a predetermined profile, the operating mode of the surface infrastructure is determined by a series of calculations and necessary decisions are made for the modernization of site facilities or laping. In the case of working with the integrated model, all calculations are performed together, and the mutual influence of the hydrodynamic modeling (HDM) and the infrastructure on each other is determined.

The integrated model is controlled by monitoring each well. Two kinds of controlare used:

- work at maximum potential(monitoring for bottomhole pressure, usually set to the minimum acceptable value, agreed with the developers);

- $\quad$ work with a control on the selection of liquids;

As a boundary condition on the surface, the pressure at the entrance to the BPS is established. Thus, the software first calculates production for each well for specified conditions, then determines the wellhead pressures to ensure that the fluid is pumped to the BPS, then the ESP speed is selected, or the type of ESP and the number of steps is determined to ensure a given sampling and wellhead pressure. The final stage is the final calculation, where the newly installed ESP model or previously set, but with the specified speed value, has already been taken into account in the model and it is required to verify the correctness of calculations at the previous stages and obtain the values of pressures and other parameters of the operation mode of the collection system.

This calculation procedure allows to extend the effect of hydraulic resistances (including losses on Free Water Knock Out unit (FWKO) and pressure on the BPS for each well, and also to calculate the neces sary changes in downhole equipment in order to overcome losses in the systemand ensure target production.

Thus, the production profile automatically adapts to the change in the surface infrastructure and it becomes possible to solve tasks both for reengineering and optimization of production.

The management of the reservoir-pressure maintenance (RPM) system is implemented as follows: RPM wells are operated under group control, providing compensation of $105 \%$ (conservative estimate). The bottomhole pressures of the injection wells are set taking into account the last technological mode of operation and are further increased taking into account the possibilities to provide wellhead pressure in the PPD.

To perform the calculation of the maximum production potential of the well, work with a bottomhole pressure control of $60 \mathrm{~b}$ is assumed.

\section{CONCLUSIONS}

The integrated field model based on neural networks implemented in the software under consideration extends the 
conventional approach of reengineering the surface infrastructure, where the determining factor is the forecasted production profile for the field. The integrated model allows to adjust the production profile and visually demonstrates the effects of using various infrastructural solutions for expanding the oil collection capacity, reducing well head pressure without loss in production of liquid.

A promising trend in the use of integrated models is the use of this type of calculation to manage the production of the oilfield, to calculate the consequences of temporary decommissioning of equipment, to calculate the technological regimes of the well groups needed to compens ate for the dropout. The ability to predict the long-term future of technological modes of operation of surface equipment and wells allows to reduce capital costs aimed at reserving capacities and justify these solutions by accurate calculation.

\section{ACKNOWLEDGMENT}

This paper is published due the financial support of the Russian Science Foundation (RSF) via the grant No 15-1900196.

\section{REFERENCES}

[1] Ya.S. Korovin., M.G. Tkachenko, S.V. Kononov, Oilfield equipment's state diagnostics on the basis of data mining technologies (In Russ.), Neftyanoekhozyaystvo = Oil Industry, no. 9, pp. 116-118,2012.

[2] Ya.S. Korovin., M.G. Tkachenko, Method of calculation of coordinates of height of products in system of contactless definition of raznovysotnosti t vs of the active zone of the reactor(In Russ.), Izvestiya Yuzhnogo Federal'nogo Universiteta. Tekhnicheskie nauki = Izvestiya SFedU. Engineering sciences, V. 113, no. 12, pp. 172-178, 2010.

[3] Ya.S. Korovin., M.V. Khisamut dinov, M.G. Tkachenko, Forecasting of oilfield equipment work conditions with the application of evolutionary algorithms and artificial neural networks (In Russ.), Neftyanoe khozyaystvo= Oil Industry, no. 12, pp. 128-133, 2013.

[4] Iakov S. Korovin, Igor A. Kalyaev, Modern Decision Support Systems in Oil Industry: Types, Approaches and Applications, International Conference on Test, Measurement and Comput ational Methods (TMCM) 2015, Chiang Mai, THAILAND; Advances in Computer Science Research. ISBN 978-94-6252-132-2. ISSN 2352-538x, pp. 141-144.

[5] Iakov S. Korovin, The Importance of New Approaches Development and their Implementation in the Oil and Gas Industry in Russian Federation-the Current Situation Analysis, International Conference on Advances in Energy, Environment and Chemical Engineering (AEECE) 2015, Changsha, PEOPLES R CHINA; Advances in Engineering Research. ISBN: 978-94-6252-109-4. ISSN: 2352-5401. Atlantis Press, Volume 23, 2015, p.94-97.

[6] Iakov S. Korovin, Anatoly I. Kalyaev, Maksim V. Khisamutdinov, Data Mining Methods Application to the Problem of Handling Corporative Dat aset on Heavy Oil Production, International Conference on Intelligent Control and Computer Application (ICCA), Zhengzhou, PEOPLES R CHINA: JAN 16-17, 2016 Proceedings of the 2016 international conference on intelligent control and computer application, ACSRAdvances in Comptuer Science Research Volume 30, pp. 387-389.

[7] I.S. Korovin, M.V. Khisamutdinov, G. Schaefer, A. Kalyaev, Real-time diagnostics of oil production equipment using data mining, 2016 th International Conference on Informatics, Electronics and Vision (ICIEV), 2016, pp. 1196 - 1172, DOI: 10.1109/ICIEV.2016. 7760184.

[8] I.S. Korovin, M.V. Khisamutdinov, G. Schaefer, A. Kaly aev, Application of hybrid data mining methods to increase profitability of heavy oil production, 20165th International Conference on Informatics, Electronics and Vision (ICIEV), 2016, pp. 1149 - 1152, DOI: 10.1109/ICIEV.2016.7760179.V.S. Kostyshin. Simulation of operation modes of centrifugal pumps on the base of the electrohydraulic analog.Ivano-Frankovsk, - pp. 42, 2004. 